\title{
Preface: special issue MAGO 2014
}

\author{
Leocadio G. Casado ${ }^{1}$ • Eligius M. T. Hendrix ${ }^{2,3}$
}

Received: 18 October 2015 / Accepted: 21 October 2015 / Published online: 6 November 2015

C) Springer Science+Business Media New York 2015

\section{Preface}

Global Optimization Workshops are organized as light overhead meetings rather spontaneously by members of the Global Optimization scientific community. Without presidents and committees in a flat organization, its aim is to stimulate discussion between senior and junior researchers on the topic of Global Optimization in a one stream setting. The tradition continues since its first meetings in Sopron (1985 and 1990) followed by Szeged (1995), Florence (GO'99 1999), Hanmer Springs (NZ, Let's GO 2001), Santorini (2003), San José (GO05 2005), Mykonos (2007), Skukuza (SAGO, 2008), Toulouse (TOGO 2010) and Natal (Br, NAGO 2012) and was taking place in 2014 in Málaga under the name Mathematical and Applied Global Optimization (MAGO).

The organisers are from a group of researchers of the Supercomputing-Algorithms group in southern Spain. The costs were kept low due to the support of Málaga University and specifically the school of Industrial Engineering where the meeting took place. More than 40 interested researchers sent in an extended abstract, which was used as a discussion document describing a problem and/or algorithm to be deliberated during the meeting. Finally, Panos Pardalos opened the workshop with an overview of research questions that have been dealt with and topics that are still open for further research. An interesting discussion was due to the frontier question of what makes optimization problems hard to be solved from a complexity point of view. During several days all researchers presented their research questions and the corresponding challenges. Interaction and discussion were promoted by organising several

Leocadio G. Casado

leo@ual.es

Eligius M. T. Hendrix

eligius.hendrix@wur.nl

1 Departmento de Informática, Universidad de Almería, Agrifood Campus of International Excellence (ceiA3), Almería, Spain

2 Computer Architecture, Universidad de Málaga, Málaga, Spain

3 Operations Research and Logistics, Wageningen University, Wageningen, The Netherlands 
excursions after which the session ended on the beach. The editor in chief insisted to test the hypothesis that discussion on Global Optimization is stimulated by playing a soccer match against the locals.

After the workshop the participants were challenged to elaborate the reseach questions further and send in a contribution for the Journal of Global Optimization. The papers were reviewed in the usual process and finally seven manuscripts were accepted for publication in this special issue. Although stochastic algorithms and other heuristics were also discussed during the meeting, all papers in this issue deal with deterministic algorithms that guarantee to find the optimum up to a predefined accuracy.

Dag Haugland studied complexity issues of the Pooling Problem. Milan Hladic developed underestimation of Hessian matrices. Boglárka G.-Tóth and her student Kristóf Kovács studied a Stackelberg location problem to be solved rigorously by branch and bound. Andreas Lundell and coworkers extended a supporting hyperplane method for MINLP. Satafa Sanago and Frédéric Messine applied branch and bound to a topology optimization problem. Bertrand Neveu and coworkers studied how node selection strategies may influence the efficiency of branch and bound methods. Finally Boglárka G.-Tóth and coworkers showed that using regular overlapping simplices to perform branch and bound on the unit simplex may surprisingly not be necessarily worse than using a parition.

We wish the readers of this special issue to join us in the fruitful learning process caught by the magic of the so-called XII Global Optimization Workshop known as Mathematical and Applied Global Optimization (MAGO) 2014. We are convinced that the following workshop GOW 2016 in Braga (Portugal) will provide ample opportunities to discuss new challenging GO problems and deterministic and stochastic algorithms.

The editors. 